International Journal of Applied Mathematics

Volume 34 No. $5 \quad 2021,995-1012$

ISSN: $1311-1728$ (printed version); ISSN: 1314-8060 (on-line version)

doi: http://dx.doi.org/10.12732/ijam.v34i5.8

\title{
DYNAMICAL BEHAVIORS OF A NEW SIS EPIDEMIC MODEL ON SCALE-FREE NETWORKS WITH STOCHASTIC PERTURBATION
}

\author{
Meici Sun ${ }^{1}$, Qiming $\operatorname{Liu}^{2}$ \\ 1,2 Department of Scientific Culture \\ Shijiazhuang Branch \\ Army Engineering University of PLA \\ Shijiazhuang 050003, P. R. CHINA
}

\begin{abstract}
A new SIS epidemic model on scale-free networks with stochastic perturbation is considered. We deduce that dynamical behaviors of the model are related to the basic reproduction number $R_{0}$ of the corresponding deterministic model. Under the conditions that the intensities of white noises interference are sufficiently weak, the solution of the model oscillates around the disease-free equilibrium of the corresponding deterministic model when $R_{0}<1$, whereas, the disease will be persistent when $R_{0}>1$.
\end{abstract}

AMS Subject Classification: 92B05, 93E15, 60H10, 34E10

Key Words: stochastic SIS model; scale-free network; stochastic Lyapunov function; persistence

\section{Introduction}

Epidemiology is the science to investigate transmission rules of infectious diseases from the group level, so as to make measures of intervention and control.

Received: March 25, 2021

(C) 2021 Academic Publications

$\S_{\text {Correspondence author }}$ 
It depends not only on the biological features of infections but also on the contact patterns between populations. With scale-free network theory by Barabási and Albert [1] developing and maturing, it has been considered as a new and effective tool to depict and study various complex architectures in nature and human society. It is more practical to study the spread mechanism of infectious diseases on scale-free networks.

Pioneering work in this area was carried out by Pastor-Satorras and Vespignani $[2,3]$. They introduced the SIS model on scale-free networks and showed that the spread of infections is tremendously strengthened on scale-free networks. Following the work, more and more models, for example, SI [4], SIR [5], SIRS $[6,7]$ and SEIRS [8], on scale-free networks were formulated successively. Notably, a new SIS model with birth and death on scale-free networks was proposed by Zhang and Jin [9]. As mentioned in the paper, the nodes of complex network have three states: vacant state, susceptible individual occupation and infected individual occupation, and each node is either empty or occupied by only one individual. The disease transmission diagram is depicted in Fig. 1. Let $S_{k}(t)$ and $I_{k}(t)$ denote the relative densities of healthy and infected nodes with degree $k$ at time $t$, respectively, where $k=1,2, \cdots, n$ in which $n$ is the maximum connectivity of any node. Then the equations for the densities $S_{k}(t)$ and $I_{k}(t)$, at the mean-field level, can be expressed as

$$
\begin{aligned}
& \frac{\mathrm{d} S_{k}(t)}{\mathrm{d} t}=b\left(1-S_{k}(t)-I_{k}(t)\right)-\lambda k S_{k}(t) \Theta(t)-d S_{k}(t)+\gamma I_{k}(t), \\
& \frac{\mathrm{d} I_{k}(t)}{\mathrm{d} t}=\lambda k S_{k}(t) \Theta(t)-\gamma I_{k}(t)-\mu I_{k}(t),
\end{aligned}
$$

where $\Theta=1 /\langle k\rangle \sum_{j=1}^{n} \varphi(j) P(j) I_{j}, \varphi(j)$ denotes an infected node, with degree $j$, occupied edges which can transmit the disease. The parameters in system (1) are summarized as follows:

$b$ : birth rate of empty nodes to healthy individuals,

$\lambda$ : transmission coefficient of the disease between susceptible nodes and infected nodes,

$d$ : death rate due to natural death,

$\gamma$; recovery rate of infected individuals,

$\mu$ : death rate due to the infectious disease and natural death.

Denote $\beta_{k j}=\frac{1}{\langle k\rangle} \lambda k \varphi(j) P(j)$, then system (1) can be rewritten as 


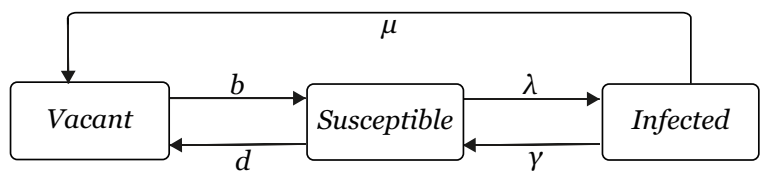

Fig. 1: The flow diagram of the new SIS model

$$
\begin{aligned}
\mathrm{d} S_{k} & =\left[b\left(1-S_{k}-I_{k}\right)-\sum_{j=1}^{n} \beta_{k j} S_{k} I_{j}-d S_{k}+\gamma I_{k}\right] \mathrm{d} t \\
\mathrm{~d} I_{k} & =\left[\sum_{j=1}^{n} \beta_{k j} S_{k} I_{j}-\gamma I_{k}-\mu I_{k}\right] \mathrm{d} t .
\end{aligned}
$$

For system (2), according to the results given in Ref.[9], there always exists a disease-free equilibrium $E^{0}=\left(\frac{b}{b+d}, 0, \cdots, \frac{b}{b+d}, 0\right)$. If $R_{0}<1, E^{0}$ is globally asymptotically stable in $D$. Otherwise, $E^{0}$ is unstable and it has a unique endemic equilibrium $E^{*}=\left(S_{1}^{*}, I_{1}^{*}, \cdots, S_{n}^{*}, I_{n}^{*}\right)$, which is globally asymptotically stable under the condition $\gamma>b$. Here

$$
\begin{gathered}
D=\left\{\left(S_{1}, I_{1}, \cdots, S_{n}, I_{n}\right) \in \mathbb{R}_{+}^{2 n} \mid 0<S_{k}+I_{k}<\frac{b}{b+d}, k=1, \cdots, n\right\}, \\
M_{0}=\left(\frac{\beta_{k j} b}{(\gamma+\mu)(b+d)}\right)_{n \times n}, \quad R_{0}=\rho\left(M_{0}\right),
\end{gathered}
$$

and $\rho\left(M_{0}\right)$ is the spectral radius of $M_{0}$.

However, there are uncertainty and random environment in nature, which inevitably affect the dynamical behaviors of epidemic spreading. Thus deterministic models have some limitations in describing the spread of diseases. The environmental noise in the ecosystem can be molded by Brownian motion. It is more suitable to describe the disease spreading with stochastic differential equations with Brownian motion as noise, i.e., stochastic epidemic model. In recent years, many scholars have focused on stochastic epidemic models on complex networks and a lot of results have emerged [10, 11, 12].

Motivated by the works in $[9,10,11,12]$, we will consider the following new 
SIS model with stochastic perturbation

$$
\begin{aligned}
\mathrm{d} S_{k} & =\left[b\left(1-S_{k}-I_{k}\right)-\sum_{j=1}^{n} \beta_{k j} S_{k} I_{j}-d S_{k}+\gamma I_{k}\right] \mathrm{d} t+\sigma_{k 1} S_{k} \mathrm{~d} W_{k 1}(t), \\
\mathrm{d} I_{k} & =\left[\sum_{j=1}^{n} \beta_{k j} S_{k} I_{j}-\gamma I_{k}-\mu I_{k}\right] \mathrm{d} t+\sigma_{k 2} I_{k} \mathrm{~d} W_{k 2}(t),
\end{aligned}
$$

where $W_{k j}(t), \sigma_{k j}^{2}, k=1,2, \cdots, n, j=1,2$ represent independent standard Brownian motions and the intensities of white noises interference respectively.

The rest of this paper is organized as follows. The existence and uniqueness of positive solution of system(3) are discussed in the next section. In Section 3 , we analyze the asymptotic behavior around the disease-free equilibrium of corresponding deterministic system (2). In Section 4, we study the dynamics of system (3) around the endemic equilibrium of system (2). Numerical simulations are given to demonstrate the main results and conclusions are drawn in Section 5 and Section 6.

\section{Existence and uniqueness of positive solution}

For any biological system, in order to study dynamical behaviors, whether it has a global positive solution is the primary issue. In this section, we will discuss this aspect by the Lyapunov analysis method. The suitable parameters in Lyapunov function are chosen by graph theory. The Laplacian matrix of a weighted digraph $(\Im, A)$ is denoted by $L_{A}$. For convenience, we denote the solution of system (3) by $X(t)=\left(S_{1}(t), I_{1}(t), \cdots, S_{n}(t), I_{n}(t)\right)$.

Theorem 1. For any initial value $X(0) \in \mathbb{R}_{+}^{2 n}$, there exists a unique solution to system (3) for all $t \geq 0$ and the solution remains in $\mathbb{R}_{+}^{2 n}$ almost surely for all times.

Proof. Since the coefficients of system (3) are locally Lipschitz continuous, for any initial value $X(0) \in \mathbb{R}_{+}^{2 n}$, there exists a unique local solution on $t \in$ $\left[0, \tau_{e}\right)$, where $\tau_{e}$ is the explosion time. To show that the solution is global, we only need to show $\tau_{e}=\infty$ a.s.. Let $m_{0}$ be a sufficiently large integer such that $\frac{1}{m_{0}}<S_{k}(0)<m_{0}, \frac{1}{m_{0}}<I_{k}(0)<m_{0}$ for $k=1, \cdots, n$. For each integer $m>m_{0}$, 
define the stopping time as

$$
\begin{gathered}
\tau_{m}=\inf \left\{t \in\left[0, \tau_{e}\right): \min \left\{S_{k}(t), I_{k}(t), k=1, \cdots, n\right\} \leq \frac{1}{m}\right. \\
\text { or } \left.\max \left\{S_{k}(t), I_{k}(t), k=1, \cdots, n\right\} \geq m\right\},
\end{gathered}
$$

where we let $\inf \emptyset=+\infty$. Obviously, $\tau_{m}$ is increasing as $m \rightarrow \infty$. Denoting $\lim _{m \rightarrow \infty} \tau_{m}=\tau_{\infty}$, we can obtain that $\tau_{\infty} \leq \tau_{e}$ a.s.. Thus, we only need to show that $\tau_{\infty}=\infty$ a.s.. Next, We prove it by contradiction. If $\tau_{\infty}<\infty$ a.s., there would exist a pair of constants $T>0$ and $\epsilon \in(0,1)$ such that $P\left(\tau_{\infty} \leq T\right)>\epsilon$. Then there is an integer $m_{1}>m_{0}$ such that

$$
P\left(\tau_{m} \leq T\right)>\epsilon, \quad \text { for } \forall m \geq m_{1} .
$$

Let us define a $C^{2}$-function $V: \mathbb{R}_{+}^{2 n} \rightarrow \mathbb{R}_{+}$as

$$
V(X(t))=\sum_{k=1}^{n}\left[\left(S_{k}-a q_{k}-a q_{k} \ln \frac{S_{k}}{a q_{k}}\right)+\left(I_{k}-1-\ln I_{k}\right)\right],
$$

where $q_{k}$ is the cofactor of the $k$-th diagonal entry of $L_{B}, B=\left(\beta_{k j}\right)_{n \times n}$, and $a$ is a positive constant to be determined. By Itô's formula, a direct calculation yields

$$
\mathrm{d} V=L V \mathrm{~d} t+\sum_{k=1}^{n}\left[\sigma_{k 1}\left(S_{k}-a q_{k}\right) \mathrm{d} W_{k 1}(t)+\sigma_{k 2}\left(I_{k}-1\right) \mathrm{d} W_{k 2}(t)\right]
$$

where

$$
\begin{aligned}
& L V=\sum_{k=1}^{n}\left[\left(1-\frac{a q_{k}}{S_{k}}\right)\left(b\left(1-S_{k}-I_{k}\right)-\sum_{j=1}^{n} \beta_{k j} S_{k} I_{j}-d S_{k}+\gamma I_{k}\right)\right.\left.\left(1-\frac{1}{I_{k}}\right)\left(\sum_{j=1}^{n} \beta_{k j} S_{k} I_{j}-\gamma I_{k}-\mu I_{k}\right)+\frac{1}{2}\left(a q_{k} \sigma_{k 1}^{2}+\sigma_{k 2}^{2}\right)\right] \\
& \leq \sum_{k=1}^{n}\left[a q_{k}(b+d)+a q_{k} \sum_{j=1}^{n} \beta_{k j} I_{j}+\gamma+\mu+b-(b+\mu) I_{k}\right. \\
&\left.\quad+\frac{1}{2}\left(a q_{k} \sigma_{k 1}^{2}+\sigma_{k 2}^{2}\right)\right] \\
&=\sum_{k=1}^{n}\left[a q_{k}(b+d)+\gamma+\mu+b-\left(b+\mu-a q_{k} \sum_{j=1}^{n} \beta_{k j}\right) I_{k}\right.
\end{aligned}
$$




$$
\left.+\frac{1}{2}\left(a q_{k} \sigma_{k 1}^{2}+\sigma_{k 2}^{2}\right)\right]
$$

The last equality above is based on $\sum_{k, j=1}^{n} q_{k} \beta_{k j} I_{j}=\sum_{k, j=1}^{n} q_{k} \beta_{k j} I_{k}$ from Theorem 2.3 in Ref. [13]. Choose $a=\min _{1 \leq k \leq n}\left\{\frac{b+u}{q_{k} \sum_{j=1}^{n} \beta_{k j}}\right\}$ such that $b+\mu-a q_{k} \sum_{j=1}^{n} \beta_{k j} \geq 0$. Therefore,

$$
\mathrm{d} V \leq K \mathrm{~d} t+\sum_{k=1}^{n}\left[\sigma_{k 1}\left(S_{k}-a q_{k}\right) \mathrm{d} W_{k 1}(t)+\sigma_{k 2}\left(I_{k}-1\right) \mathrm{d} W_{k 2}(t)\right],
$$

where $K=\sum_{k=1}^{n}\left[a q_{k}(b+d)+\gamma+\mu+b+\frac{1}{2}\left(a q_{k} \sigma_{k 1}^{2}+\sigma_{k 2}^{2}\right)\right]$. Integrating both sides of (5) from 0 to $\tau_{m} \wedge T$ and then taking the expectations, we obtain that

$$
\begin{aligned}
V(X(0))+K T & \geq E V\left(X\left(\tau_{m} \wedge T\right)\right) \\
& \geq E\left[1_{\left\{\tau_{m} \leq T\right\}} V\left(X\left(\tau_{m}\right)\right)\right],
\end{aligned}
$$

where $1_{\left\{\tau_{m} \leq T\right\}}$ is the indicator function of $\left\{\tau_{m} \leq T\right\}$. For any $\omega \in\left\{\tau_{m} \leq\right.$ $T\}$, where $m>m_{1}$, there exists at least one of $S_{k}\left(\tau_{m}, \omega\right)$ and $I_{k}\left(\tau_{m}, \omega\right), k=$ $1,2, \cdots, n$, equaling $\frac{1}{m}$ or $m$, then

$$
\begin{aligned}
V\left(X\left(\tau_{m}\right)\right) \geq & \min _{0 \leq k \leq n}\left(m-a q_{k}-a q_{k} \ln \frac{m}{a q_{k}}\right) \\
& \wedge \min _{0 \leq k \leq n}\left(\frac{1}{m}-a q_{k}+a q_{k} \ln \left(a q_{k} m\right)\right),
\end{aligned}
$$

where we define $q_{0}=\frac{1}{a}$. From (4), (6) and (7), it follows that

$$
\begin{aligned}
V(X(0))+K T \geq \epsilon & {\left[\min _{0 \leq k \leq n}\left(m-a q_{k}-a q_{k} \ln \frac{m}{a q_{k}}\right)\right.} \\
& \left.\wedge \min _{0 \leq k \leq n}\left(\frac{1}{m}-a q_{k}+a q_{k} \ln \left(a q_{k} m\right)\right)\right] .
\end{aligned}
$$

Letting $m \rightarrow \infty$, yields $\infty>V(X(0))+K T \geq \infty$, which is a contradiction. Thus the theorem is proved.

\section{Asymptotic Behavior around the Disease-free Equilibrium}

In Introduction, we mention that $E^{0}$ is the disease-free equilibrium of deterministic system (2), which is globally stable when $R_{0}<1$. It means that the 
disease will die out after some period of time. Hence, it is meaningful to study the disease-free equilibrium. For system (3), however, there is no disease-free equilibrium. Thus we will study the asymptotic behavior of system (3) around $E^{0}$.

Theorem 2. If $R_{0}<1, \gamma>b$ and

$$
\sigma_{k 1}^{2} \leq b+d, \quad \sigma_{k 2}^{2} \leq 2(b+\mu)
$$

then for any initial value $X(0) \in \mathbb{R}_{+}^{2 n}$, the solution of system (3) has the property

$$
\begin{gathered}
\limsup _{t \rightarrow \infty} \frac{1}{t} \sum_{k=1}^{n} E \int_{0}^{t}\left[\left(S_{k}(u)-\frac{b}{b+d}\right)^{2}+I_{k}^{2}(u)\right] \mathrm{d} u \\
\leq \sum_{k=1}^{n} \frac{\left(a_{k}+c_{k}\right)}{K_{1}}\left(\frac{b}{b+d}\right)^{2} \sigma_{k 1}^{2},
\end{gathered}
$$

where

$$
K_{1}=\min _{k=1, \cdots, n}\left\{\left(a_{k}+c_{k}\right)\left(b+d-\sigma_{k 1}^{2}\right), a_{k}\left(b+\mu-\frac{\sigma_{k 2}^{2}}{2}\right)\right\},
$$

and $a_{k}, c_{k}, k=1,2, \cdots, n$ are positive constants defined as in the proof.

Proof. Let $s_{k}=S_{k}-\frac{b}{b+d}, i_{k}=I_{k}$, then $s_{k} \geq-\frac{b}{b+d}, i_{k} \geq 0$. For system (3), it can be rewritten as

$$
\begin{aligned}
\mathrm{d} s_{k}(t)= & {\left[-(b+d) s_{k}-\sum_{j=1}^{n} \beta_{k j}\left(s_{k}+\frac{b}{b+d}\right) i_{j}+(\gamma-b) i_{k}\right] \mathrm{d} t } \\
& +\sigma_{k 1}\left(s_{k}+\frac{b}{b+d}\right) \mathrm{d} W_{k 1}(t), \\
\mathrm{d} i_{k}(t)= & {\left[\sum_{j=1}^{n} \beta_{k j}\left(s_{k}+\frac{b}{b+d}\right) i_{j}-(\gamma+\mu) i_{k}\right] \mathrm{d} t+\sigma_{k 2} i_{k} \mathrm{~d} W_{k 2}(t) . }
\end{aligned}
$$

Since all elements of $M_{0}=\frac{b}{(\gamma+\mu)(b+d)}\left(\beta_{k j}\right)_{n \times n}$ are positive, then $M_{0}$ is irreducible. By Lemma 2.1 of Guo et al. [14], there exists a positive eigenvector $\omega=\left(\omega_{1}, \omega_{2}, \cdots, \omega_{n}\right)$ of $M_{0}$ corresponding to $\rho\left(M_{0}\right)$, namely that

$$
\left(\omega_{1}, \omega_{2}, \cdots, \omega_{n}\right) M_{0}=\left(\omega_{1}, \omega_{2}, \cdots, \omega_{n}\right) \rho\left(M_{0}\right) .
$$

Let $x(t)=\left(s_{1}(t), i_{1}(t), \cdots, s_{n}(t), i_{n}(t)\right)$ and define the Lyapunov function as 


$$
V(x(t))=\frac{1}{2} \sum_{k=1}^{n} a_{k}\left(s_{k}+i_{k}\right)^{2}+\frac{1}{2} \sum_{k=1}^{n} c_{k} s_{k}^{2}+\sum_{k=1}^{n} \frac{\omega_{k}}{\gamma+\mu} i_{k},
$$

where $a_{k}, c_{k}, k=1,2 \cdots, n$ are undetermined positive constants. By Itô's formula, a direct calculation yields

$$
\begin{aligned}
\mathrm{d} V= & L V \mathrm{~d} t+\sum_{k=1}^{n}\left[a_{k}\left(s_{k}+i_{k}\right)+c_{k} s_{k}\right] \sigma_{k 1}\left(s_{k}+\frac{b}{b+d}\right) \mathrm{d} W_{k 1}(t) \\
& +\sum_{k=1}^{n}\left[a_{k}\left(s_{k}+i_{k}\right)+\frac{\omega_{k}}{\gamma+\mu}\right] \sigma_{k 2} i_{k} \mathrm{~d} W_{k 2}(t),
\end{aligned}
$$

where

$$
\begin{aligned}
L V= & \sum_{k=1}^{n} a_{k}\left(s_{k}+i_{k}\right)\left[-(b+d) s_{k}-(b+\mu) i_{k}\right] \\
& +\sum_{k=1}^{n} c_{k} s_{k}\left[-(b+d) s_{k}-\sum_{j=1}^{n} \beta_{k j}\left(s_{k}+\frac{b}{b+d}\right) i_{j}+(\gamma-b) i_{k}\right] \\
& +\frac{1}{2} \sum_{k=1}^{n}\left(a_{k}+c_{k}\right) \sigma_{k 1}^{2}\left(s_{k}+\frac{b}{b+d}\right)^{2}+\frac{1}{2} \sum_{k=1}^{n} a_{k} \sigma_{k 2}^{2} i_{k}^{2} \\
& +\sum_{k=1}^{n} \frac{\omega_{k}}{\gamma+\mu}\left[\sum_{j=1}^{n} \beta_{k j}\left(s_{k}+\frac{b}{b+d}\right) i_{j}-(\gamma+\mu) i_{k}\right] \\
\leq & -\sum_{k=1}^{n}\left[\left(a_{k}+c_{k}\right)\left(b+d-\sigma_{k 1}^{2}\right) s_{k}^{2}+a_{k}\left(b+\mu-\frac{\sigma_{k 2}^{2}}{2}\right) i_{k}^{2}\right] \\
& +\sum_{k=1}^{n}\left[c_{k}(\gamma-b)-a_{k}(2 b+d+\mu)\right] s_{k} i_{k} \\
& -\sum_{k=1}^{n} \sum_{j=1}^{n} \frac{b c_{k} \beta_{k j}}{b+d} s_{k} i_{j}+\sum_{k=1}^{n}\left(a_{k}+c_{k}\right)\left(\frac{b}{b+d}\right)^{2} \sigma_{k 1}^{2} \\
& +\sum_{k=1}^{n} \sum_{j=1}^{n} \frac{\omega_{k} \beta_{k j}}{\gamma+\mu} s_{k} i_{j}+\sum_{k=1}^{n} \sum_{j=1}^{n} \frac{b \omega_{k} \beta_{k j}}{(b+d)(\gamma+\mu)} i_{j}-\sum_{k=1}^{n} \omega_{k} i_{k} .
\end{aligned}
$$

The inequality above is using $(x+y)^{2} \leq 2\left(x^{2}+y^{2}\right)$ and the fact $i_{k}>0$. Let 
$\omega=\left(\omega_{1}, \cdots, \omega_{n}\right), i=\left(i_{1}, \cdots, i_{n}\right)^{\top}$, and we note that

$$
\begin{aligned}
& \sum_{k=1}^{n} \sum_{j=1}^{n} \frac{b \omega_{k} \beta_{k j}}{(b+d)(\gamma+\mu)} i_{j}-\sum_{k=1}^{n} \omega_{k} i_{k} \\
= & \omega M_{0} i-\omega i=\omega \rho\left(M_{0}\right) i-\omega i \\
= & \left(R_{0}-1\right) \sum_{k=1}^{n} \omega_{k} i_{k}<0,
\end{aligned}
$$

due to $R_{0}<1$ and $i_{k}>0$. Since $\gamma>b$, we can choose $c_{k}=\frac{\omega_{k}(b+d)}{b(\gamma+\mu)}, a_{k}=$ $\frac{c_{k}(\gamma-b)}{2 b+d+\mu}>0$ such that $\frac{\omega_{k} \beta_{k j}}{\gamma+\mu}-c_{k} \beta_{k j} \frac{b}{b+d}=0$ and $c_{k}(\gamma-b)-a_{k}(2 b+d+\mu)=0$, which together with (13) yield

$$
\begin{aligned}
L V \leq & -\sum_{k=1}^{n}\left[\left(a_{k}+c_{k}\right)\left(b+d-\sigma_{k 1}^{2}\right) s_{k}^{2}+a_{k}\left(b+\mu-\frac{\sigma_{k 2}^{2}}{2}\right) i_{k}^{2}\right] \\
& +\sum_{k=1}^{n}\left(a_{k}+c_{k}\right)\left(\frac{b}{b+d}\right)^{2} \sigma_{k 1}^{2} \\
:= & F(t)
\end{aligned}
$$

Substituting (14) into (12) and integrating both side from 0 to $t$, we obtain that

$$
0 \leq E V(x(t)) \leq V(x(0))+E \int_{0}^{t} F(u) \mathrm{d} u
$$

where $E$ denotes expectation operator. It implies that

$$
\begin{aligned}
& E \int_{0}^{t} \sum_{k=1}^{n}\left[\left(a_{k}+c_{k}\right)\left(b+d-\sigma_{k 1}^{2}\right) s_{k}^{2}(u)+a_{k}\left(b+\mu-\frac{\sigma_{k 2}^{2}}{2}\right) i_{k}^{2}(u)\right] \mathrm{d} u \\
& \leq V(x(0))+\sum_{k=1}^{n}\left(a_{k}+c_{k}\right)\left(\frac{b}{b+d}\right)^{2} \sigma_{k 1}^{2} t .
\end{aligned}
$$

Hence,

$$
\begin{gathered}
\limsup _{t \rightarrow \infty} \frac{1}{t} E \int_{0}^{t} \sum_{k=1}^{n}\left[\left(a_{k}+c_{k}\right)\left(b+d-\sigma_{k 1}^{2}\right)\left(S_{k}(u)-\frac{b}{b+d}\right)^{2}\right. \\
\left.\quad+a_{k}\left(b+\mu-\frac{\sigma_{k 2}^{2}}{2}\right) I_{k}^{2}(u)\right] \mathrm{d} u \\
\leq \sum_{k=1}^{n}\left(a_{k}+c_{k}\right)\left(\frac{b}{b+d}\right)^{2} \sigma_{k 1}^{2} .
\end{gathered}
$$


Let $K_{1}=\min \left\{\left(a_{k}+c_{k}\right)\left(b+d-\sigma_{k 1}^{2}\right), a_{k}\left(b+\mu-\sigma_{k 2}^{2} / 2\right)\right\}$, then the proof is completed.

Remark 3. Under the conditions of Theorem 2, the mean square distance between the solution of system (3) and the disease-free equilibrium $E^{0}$ of deterministic system (2) in time average is no more than a value which is related to $\sigma_{k 1}^{2}$ and $\sigma_{k 2}^{2}$. It is concluded that the solution of system (3) oscillates around $E^{0}$ and the amplitude depends on the intensities of white noises interference.

Besides, $E^{0}$ is the disease-free equilibrium of system (3), if $\sigma_{k 1}=0, k=$ $1,2, \cdots n$. Moreover, $E^{0}$ is also globally asymptotically stable, because

$$
L V \leq-\sum_{k=1}^{n}\left[\left(a_{k}+c_{k}\right)(b+d) s_{k}^{2}+a_{k}\left(b+\mu-\frac{\sigma_{k 2}^{2}}{2}\right) i_{k}^{2}\right] \leq 0
$$

from the proof of Theorem 2 .

\section{The dynamic of system (3) around $E^{*}$}

For deterministic system (2), if $R_{0}>1$, there exists an endemic equilibrium $E^{*}$, which is global stable when $\gamma>b$. It is revealed that the disease will prevail in a population. Obviously, there is no endemic equilibrium of stochastic system (3). In this section, we will discuss dynamical behaviors of system (3) around the endemic equilibrium $E^{*}$ of system (2).

Theorem 4. If $R_{0}>1, \gamma>b$, and

$$
\sigma_{k 1}^{2} \leq b+d, \quad \sigma_{k 2}^{2} \leq b+\mu,
$$

then for any initial value $X(0) \in \mathbb{R}_{+}^{2 n}$, the solution of system (3) has the property

$$
\begin{aligned}
\limsup _{t \rightarrow \infty} & \frac{1}{t} \sum_{k=1}^{n} E \int_{0}^{t}\left[\left(S_{k}(u)-S_{k}^{*}\right)^{2}+\left(I_{k}(u)-I_{k}^{*}\right)^{2}\right] \mathrm{d} u \\
\leq & \frac{1}{K_{2}} \sum_{k=1}^{n}\left[\left(\frac{\bar{c}_{k}}{2} S_{k}^{*}+p_{k} S_{k}^{* 2}\right) \sigma_{k 1}^{2}+\left(\frac{\bar{c}_{k}}{2} I_{k}^{*}+p_{k} I_{k}^{* 2}\right) \sigma_{k 2}^{2}\right],
\end{aligned}
$$

where

$$
K_{2}=\min _{k=1, \cdots, n}\left\{p_{k}\left(b+d-\sigma_{k 1}^{2}\right), p_{k}\left(b+\mu-\sigma_{k 2}^{2}\right)\right\},
$$


and $\bar{c}_{k}$ is the cofactor of the $k$-th diagonal entry of $L_{\bar{B}}, k=1, \cdots, n, \bar{B}=$ $\left(\bar{\beta}_{k j}\right)_{n \times n}=\left(\tilde{\beta}_{k j} S_{k}^{*} I_{j}^{*}\right)_{n \times n}$, and $p_{k}, k=1,2, \cdots, n$ are positive constants defined as in the proof.

Proof. Firstly, for system (3), we use the identical technique of Kuniya et al. [15] for a multi-group SIS model to remove the terms $(\gamma-b) I_{k}$ from the Lyapunov functionals of $\mathrm{d} S_{k}$ to that of $\mathrm{d} I_{k}$. For $k, j=1,2 \cdots, n$, let

$$
\tilde{\beta}_{k k}=\beta_{k k}-\frac{\gamma-b}{S_{k}^{*}}, \quad \tilde{\beta}_{k j}=\beta_{k j} \quad k \neq j .
$$

The elements of endemic equilibrium $E^{*}$ satisfy

$$
b=\sum_{j=1}^{n} \tilde{\beta}_{k j} S_{k}^{*} I_{j}^{*}+(b+d) S_{k}^{*}, \quad \sum_{j=1}^{n} \tilde{\beta}_{k j} S_{k}^{*} I_{j}^{*}=(b+\mu) I_{k}^{*} .
$$

Substituting (15) and (16) into system (3), it follows that

$$
\begin{aligned}
\mathrm{d} S_{k}(t)=[ & -\sum_{j=1}^{n} \tilde{\beta}_{k j}\left(S_{k} I_{j}-S_{k}^{*} I_{j}^{*}\right)-(b+d)\left(S_{k}-S_{k}^{*}\right) \\
& \left.+(\gamma-b) I_{k}\left(1-\frac{S_{k}}{S_{k}^{*}}\right)\right] \mathrm{d} t+\sigma_{k 1} S_{k} \mathrm{~d} W_{k 1}(t), \\
\mathrm{d} I_{k}(t)=\left[\sum_{j=1}^{n} \tilde{\beta}_{k j}\left(S_{k} I_{j}-\frac{S_{k}^{*} I_{j}^{*} I_{k}}{I_{k}^{*}}\right)-(\gamma-b) I_{k}\left(1-\frac{S_{k}}{S_{k}^{*}}\right)\right] \mathrm{d} t & \\
& +\sigma_{k 2} I_{k} \mathrm{~d} W_{k 2}(t) .
\end{aligned}
$$

Define a $C^{2}$-function $V: \mathbb{R}_{+}^{2 n} \rightarrow \mathbb{R}^{+}$as

$$
\begin{aligned}
V(X(t)) & =\frac{1}{2} \sum_{k=1}^{n} p_{k}\left(S_{k}-S_{k}^{*}+I_{k}-I_{k}^{*}\right)^{2} \\
& +\sum_{k=1}^{n} \bar{c}_{k}\left(S_{k}-S_{k}^{*}-S_{k}^{*} \ln \frac{S_{k}}{S_{k}^{*}}+I_{k}-I_{k}^{*}-I_{k}^{*} \ln \frac{I_{k}}{I_{k}^{*}}\right) \\
& :=V_{1}+V_{2},
\end{aligned}
$$

where $p_{k}, k=1, \cdots, n$ are positive constants to be determined. The Matrix $\bar{B}$ is irreducible from the irreducibility of matrix $\left(\beta_{k j}\right)_{n \times n}$. It is follows that $\bar{c}_{k}>0, k=1,2, \cdots, n$ by the property (1) of Theorem 2.3 in Li et al. [13]. Then $V$ is positive definite. Let $L$ be the generating operator of system (17). Then one has 


$$
\begin{aligned}
L V_{1}= & \sum_{k=1}^{n} p_{k}\left(S_{k}-S_{k}^{*}+I_{k}-I_{k}^{*}\right)\left[b-(b+d) S_{k}-(b+\mu) I_{k}\right] \\
& +\frac{1}{2} \sum_{k=1}^{n} p_{k}\left(\sigma_{k 1}^{2} S_{k}^{2}+\sigma_{k 2}^{2} I_{k}^{2}\right) \\
= & \sum_{k=1}^{n} p_{k}\left(S_{k}-S_{k}^{*}+I_{k}-I_{k}^{*}\right)\left[-(b+d)\left(S_{k}-S_{k}^{*}\right)-(b+\mu)\left(I_{k}-I_{k}^{*}\right)\right] \\
& +\frac{1}{2} \sum_{k=1}^{n} p_{k}\left[\sigma_{k 1}^{2}\left(S_{k}-S_{k}^{*}+S_{k}^{*}\right)^{2}+\sigma_{k 2}^{2}\left(I_{k}-I_{k}^{*}+I_{k}^{*}\right)^{2}\right] \\
\leq & \sum_{k=1}^{n} p_{k}\left[-\left(b+d-\sigma_{k 1}^{2}\right)\left(S_{k}-S_{k}^{*}\right)^{2}-\left(b+\mu-\sigma_{k 2}^{2}\right)\left(I_{k}-I_{k}^{*}\right)^{2}\right. \\
& \left.-(2 b+d+\mu)\left(S_{k}-S_{k}^{*}\right)\left(I_{k}-I_{k}^{*}\right)+\sigma_{k 1}^{2} S_{k}^{* 2}+\sigma_{k 2}^{2} I_{k}^{* 2}\right] .
\end{aligned}
$$

And

$$
\begin{aligned}
L V_{2}= & \sum_{k=1}^{n} \bar{c}_{k}\left(1-\frac{S_{k}^{*}}{S_{k}}\right)\left[-\sum_{j=1}^{n} \tilde{\beta}_{k j}\left(S_{k} I_{j}-S_{k}^{*} I_{j}^{*}\right)-(b+d)\left(S_{k}-S_{k}^{*}\right)\right. \\
& \left.+(\gamma-b) I_{k}\left(1-\frac{S_{k}}{S_{k}^{*}}\right)\right]+\sum_{k=1}^{n} \frac{\bar{c}_{k}}{2}\left(\sigma_{k 1}^{2} S_{k}^{*}+\sigma_{k 2}^{2} I_{k}^{*}\right) \\
& +\sum_{k=1}^{n} \bar{c}_{k}\left(1-\frac{I_{k}^{*}}{I_{k}}\right)\left[\sum_{j=1}^{n} \tilde{\beta}_{k j}\left(S_{k} I_{j}-\frac{S_{k}^{*} I_{j}^{*} I_{k}}{I_{k}^{*}}\right)\right. \\
& \left.-(\gamma-b) I_{k}\left(1-\frac{S_{k}}{S_{k}^{*}}\right)\right] \\
= & -\sum_{k=1}^{n} \bar{c}_{k}\left[(b+d)+(\gamma-b) \frac{I_{k}}{S_{k}^{*}}\right] \frac{\left(S_{k}-S_{k}^{*}\right)^{2}}{S_{k}}-\sum_{k=1}^{n} \sum_{j=1}^{n} \bar{c}_{k} \bar{\beta}_{k j} . \\
& {\left[\left(1-\frac{S_{k}^{*}}{S_{k}}\right)\left(\frac{S_{k} I_{j}}{S_{k}^{*} I_{j}^{*}}-1\right)-\left(1-\frac{I_{k}^{*}}{I_{k}}\right)\left(\frac{S_{k} I_{j}}{S_{k}^{*} I_{j}^{*}}-\frac{I_{k}}{I_{k}^{*}}\right)\right] } \\
& +\sum_{k=1}^{n} \bar{c}_{k}(\gamma-b)\left(S_{k}-S_{k}^{*}\right)\left(I_{k}-I_{k}^{*}\right)+\sum_{k=1}^{n} \frac{\bar{c}_{k}}{2}\left(\sigma_{k 1}^{2} S_{k}^{*}+\sigma_{k 2}^{2} I_{k}^{*}\right) \\
\leq & -\sum_{k=1}^{n} \sum_{j=1}^{n} \bar{c}_{k} \bar{\beta}_{k j}\left[g\left(\frac{I_{k}}{I_{k}^{*}}\right)-g\left(\frac{I_{j}}{I_{j}^{*}}\right)+g\left(\frac{S_{k}^{*}}{S_{k}}\right)+g\left(\frac{S_{k} I_{j} I_{k}^{*}}{S_{k}^{*} I_{j}^{*} I_{k}}\right)\right]
\end{aligned}
$$




$$
+\sum_{k=1}^{n} \bar{c}_{k}(\gamma-b)\left(S_{k}-S_{k}^{*}\right)\left(I_{k}-I_{k}^{*}\right)+\sum_{k=1}^{n} \frac{\bar{c}_{k}}{2}\left(\sigma_{k 1}^{2} S_{k}^{*}+\sigma_{k 2}^{2} I_{k}^{*}\right)
$$

where $g(a)=a-1-\ln a$. Using property (2) of Theorem 2.3 in Li et al. [13], we know that

$$
\sum_{k=1}^{n} \sum_{j=1}^{n} \bar{c}_{k} \bar{\beta}_{k j}\left(g\left(\frac{I_{k}}{I_{k}^{*}}\right)-g\left(\frac{I_{j}}{I_{j}^{*}}\right)\right)=0 .
$$

According to (18) and the fact that $g(a) \geq 0$ for $a>0$, it follows that

$$
L V_{2} \leq \sum_{k=1}^{n} \bar{c}_{k}(\gamma-b)\left(S_{k}-S_{k}^{*}\right)\left(I_{k}-I_{k}^{*}\right)+\sum_{k=1}^{n} \frac{\bar{c}_{k}}{2}\left(\sigma_{k 1}^{2} S_{k}^{*}+\sigma_{k 2}^{2} I_{k}^{*}\right)
$$

Since $\gamma>b$, we choose $p_{k}=\frac{\bar{c}_{k}(\gamma-b)}{(2 b+d+\mu)}>0$ such that $\bar{c}_{k}(\gamma-b)-p_{k}(2 b+d+\mu)=0$. It can be obtained that

$$
\begin{aligned}
& L V=L V_{1}+L V_{2} \\
& \leq \sum_{k=1}^{n} p_{k}\left[-\left(b+d-\sigma_{k 1}^{2}\right)\left(S_{k}-S_{k}^{*}\right)^{2}-\left(b+\mu-\sigma_{k 2}^{2}\right)\left(I_{k}-I_{k}^{*}\right)^{2}\right] \\
& \quad+\sum_{k=1}^{n}\left[\left(\frac{\bar{c}_{k}}{2} S_{k}^{*}+p_{k} S_{k}^{* 2}\right) \sigma_{k 1}^{2}+\left(\frac{\bar{c}_{k}}{2} I_{k}^{*}+p_{k} I_{k}^{* 2}\right) \sigma_{k 2}^{2}\right] \\
& \quad:=G(t) .
\end{aligned}
$$

Hence,

$$
\begin{aligned}
\mathrm{d} V \leq & G(t) \mathrm{d} t+\sum_{k=1}^{n} \sigma_{k 1}\left[\bar{c}_{k}\left(S_{k}-S_{k}^{*}\right)+p_{k} S_{k}\left(S_{k}-S_{k}^{*}+I_{k}-I_{k}^{*}\right)\right] \mathrm{d} W_{k 1}(t) \\
& +\sum_{k=1}^{n} \sigma_{k 2}\left[\bar{c}_{k}\left(I_{k}-I_{k}^{*}\right)+p_{k} I_{k}\left(S_{k}-S_{k}^{*}+I_{k}-I_{k}^{*}\right)\right] \mathrm{d} W_{k 2}(t) .
\end{aligned}
$$

Integrating both sides of (19) from 0 to $t$ and taking the expectations, we have

$$
0 \leq E V(X(t)) \leq E V(X(0))+E \int_{0}^{t} G(u) \mathrm{d} u
$$


Therefore,

$$
\begin{gathered}
\limsup _{t \rightarrow \infty} \frac{1}{t} E \int_{0}^{t} \sum_{k=1}^{n} p_{k}\left[\left(b+d-\sigma_{k 1}^{2}\right)\left(S_{k}(u)-S_{k}^{*}\right)^{2}\right. \\
\left.\quad+\left(b+\mu-\sigma_{k 2}^{2}\right)\left(I_{k}(u)-I_{k}^{*}\right)^{2}\right] \mathrm{d} u \\
\leq \sum_{k=1}^{n}\left[\left(\frac{\bar{c}_{k}}{2} S_{k}^{*}+p_{k} S_{k}^{* 2}\right) \sigma_{k 1}^{2}+\left(\frac{\bar{c}_{k}}{2} I_{k}^{*}+p_{k} I_{k}^{* 2}\right) \sigma_{k 2}^{2}\right] .
\end{gathered}
$$

Let $K_{2}=\min \left\{p_{k}\left(b+d-\sigma_{k 1}^{2}\right), p_{k}\left(b+\mu-\sigma_{k 2}^{2}\right)\right\}$. This completes the proof.

Remark 5. Under the conditions of Theorem 4, the mean square distance between the solution of system (3) and the endemic equilibrium $E^{*}$ of deterministic system (2) in time average is no more than a value which is related to $\sigma_{k 1}^{2}$ and $\sigma_{k 2}^{2}$. It means that the solution of system (3) fluctuates around $E^{*}$. Therefore, It is concluded that system (3) is persistent in the mean square sense, which means that the disease is prevalent.

\section{Simulation}

In order to demonstrate the theoretical results above, we numerically simulate the solution of system (3). Consider a scale-free network whose degree distribution is $P(k)=C k^{-r}$ with $r=2.3, \sum_{k=1}^{n} P(k)=1$ and maximum connectivity of any node $n=100$. Let $b=0.05, d=0.01, \gamma=0.06, \mu=0.05$, and $\varphi(k)=a k^{\alpha} /\left(1+c k^{\alpha}\right)$ with $\alpha=0.75, a=0.8, c=0.01$. Using the Milstein's Method, the discretized difference equations of system (3) take the form

$$
\begin{gathered}
S_{k, i+1}=S_{k, i}+\Delta t\left[b\left(1-S_{k, i}-I_{k, i}\right)-\sum_{j=1}^{n} \beta_{k j} S_{k, i} I_{j, i}-d S_{k, i}+\gamma I_{k, i}\right] \\
+\sigma_{k 1} S_{k, i} \sqrt{\Delta t} \xi_{k_{1}, i}+\frac{\sigma_{k 1}^{2}}{2} S_{k, i}^{2} \Delta t\left(\xi_{k_{1}, i}^{2}-1\right), \\
I_{k, i+1}=I_{k, i}+\Delta t\left[\sum_{j=1}^{n} \beta_{k j} S_{k, i} I_{j, i}-\gamma I_{k, i}-\mu I_{k, i}\right] \\
+\sigma_{k 2} I_{k, i} \sqrt{\Delta t} \xi_{k_{2}, i}+\frac{\sigma_{k 2}^{2}}{2} I_{k, i}^{2} \Delta t\left(\xi_{k_{2}, i}^{2}-1\right),
\end{gathered}
$$



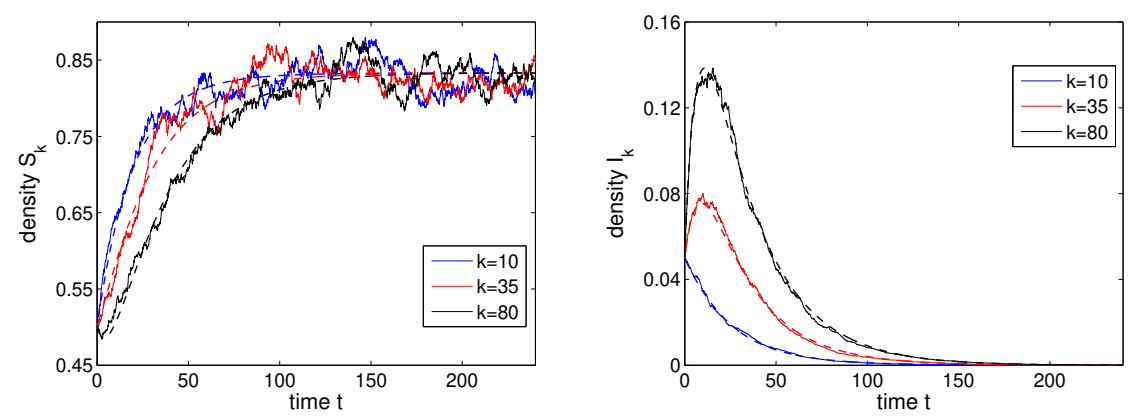

Fig. 2: The time series of system (3) with $\lambda=0.025, R_{0}=$ $0.6972, \sigma_{k 1}=0.012, \sigma_{k 2}=0.02$.
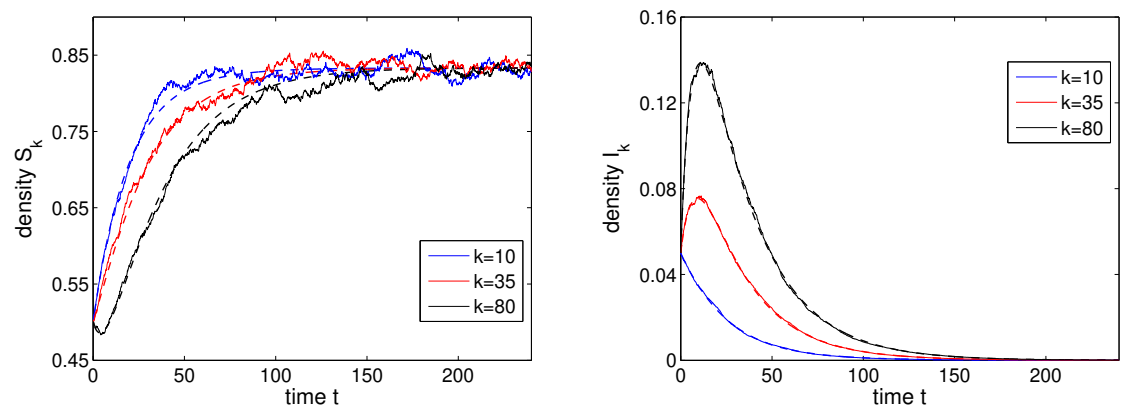

Fig. 3: The time series of system (3) with $\lambda=0.025, R_{0}=$ $0.6972, \sigma_{k 1}=0.005, \sigma_{k 2}=0.008$.

where $\xi_{k_{1}, i}, \xi_{k_{2}, i}, k_{1}, k_{2}=1,2, \cdots, n, i=1,2, \cdots, N$ are independent standard normal random variables.

First, we choose $\lambda=0.025$, then $R_{0}=0.6972<1$ by calculation. Fig. 2 and Fig. 3 (imaginary lines) depict the solution of deterministic system (2), which verify the global stability of the disease-free equilibrium $E^{0}$. From Theorem 2, the solution of system (3) oscillates around $E^{0}$, provided that the intensities are sufficiently weak. Fig. 2 and Fig. 3 (solid lines) illustrate this result under different intensities.

Next, we choose $\lambda=0.08$ such that $R_{0}=2.2311>1$. In this case, the endemic equilibrium $E^{*}$ of system (2) is globally asymptotically stable, which is shown in Fig. 4 and Fig. 5 (imaginary lines). Besides, it follows from Theorem 4 that the solution of system (3) fluctuates around $E^{*}$, which means that the disease is prevalent. Fig. 4 and Fig. 5 (solid lines) confirm this. 

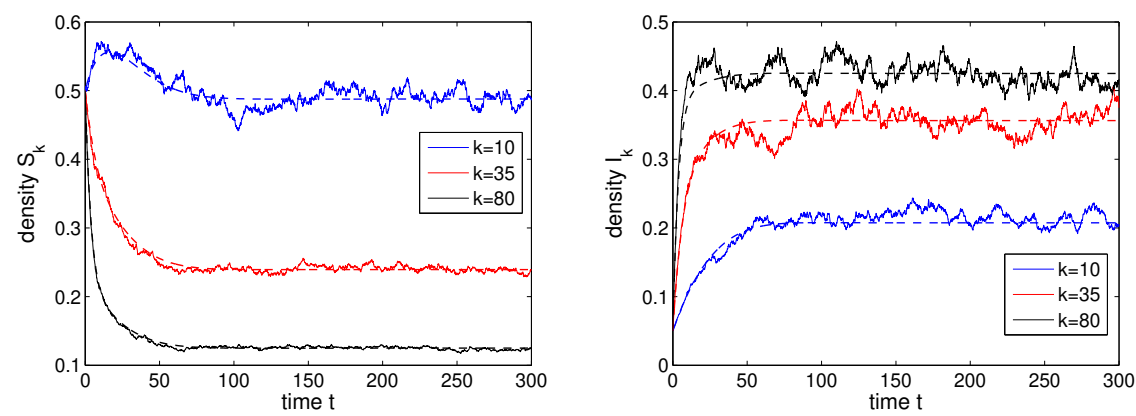

Fig. 4: The time series of system (3) with $\lambda=0.08, R_{0}=$ $2.2311, \sigma_{k 1}=0.012, \sigma_{k 2}=0.02$.
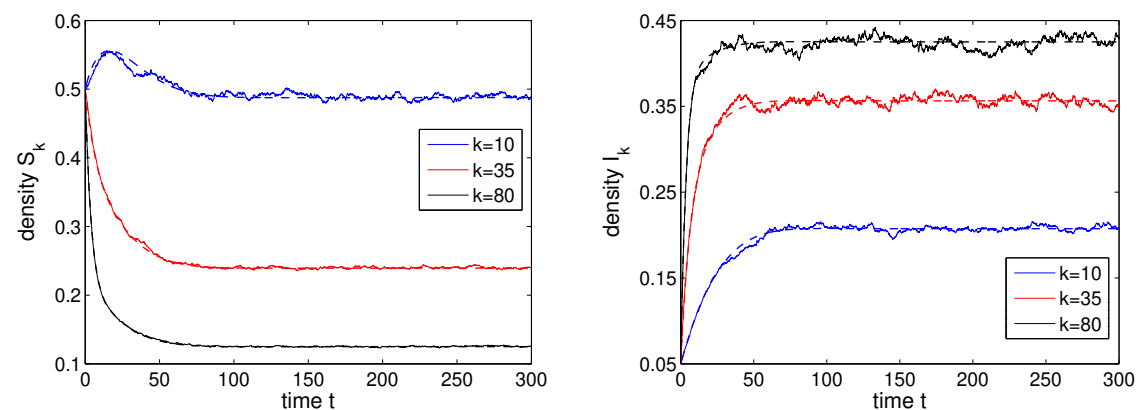

Fig. 5: The time series of system (3) with $\lambda=0.08, R_{0}=$ $2.2311, \sigma_{k 1}=0.005, \sigma_{k 2}=0.008$.

By comparing Fig. 2 and Fig. 3, the parameters are all the same but with different intensities of white noises. Specifically, $\sigma_{k 1}=0.012, \sigma_{k 2}=0.02$ in Fig. 2 and $\sigma_{k 1}=0.005, \sigma_{k 2}=0.008$ in Fig. 3. It easily can be seen that the range of oscillation around $E^{0}$ gets smaller with the intensities getting weaker. The same result is also showed up by comparing Fig. 4 and Fig. 5.

\section{Conclusion}

A new SIS epidemic model with stochastic perturbation has been proposed by stochastic differential equations to investigate the dynamical behaviors of epidemics on scale-free networks. Through theoretical analysis, we obtain that the existence and uniqueness of solution to the model. Under the conditions that the intensities of white noises interference are weak enough, the solution of 
system (3) oscillates around the disease-free equilibrium of the corresponding deterministic model (2) when $R_{0}<1$. Otherwise, the solution of system (3) fluctuates around the endemic equilibrium of model (2) when $R_{0}>1$, which means that the disease will be persistent. Moreover, the range of oscillation gets smaller with the intensities of white noises decreasing.

Because $S_{k}(t), I_{k}(t)$ denote the relative densities of the healthy and infected nodes with degree $k$, it is necessary to consider whether the solution of system (3) satisfies $S_{k}(t)+I_{k}(t) \leq 1, k=1,2, \cdots, n$. The sufficient condition for boundedness of the solution is an interesting but challenging problem.

\section{Acknowledgments}

This research was supported by the Foundation for Basic Disciplines of Army Engineering University under Grant No. KYSZJQZL2011.

\section{References}

[1] A.L. Barabási, R. Alber, Emergence of scaling in random networks, Science, 286 (1999), 509-512.

[2] R. Pastor-Satorras, A. Vespignani, Epidemic spreading in scale-free networks, Phys. Rev. Lett., 86 (2001), 3200-3203.

[3] R. Pastor-Satorras, A. Vespignani, Epidemic dynamics and endemic states in complex networks, Phys. Rev. E, 63 (2001), \# 066117.

[4] T. Zhou, J. Liu, W. Bai, G. Chen, B. Wang, Behaviors of susceptibleinfected epidemics on scale-free networks with identical infectivity, Phys. Rev. E, 74 (2006), \# 056109.

[5] Y. Moreno, R. Pastor-Satorras, A. Vespignanil, Epidemic outbreaks in complex heterogeneous networks, Eur. Phys. J. B, 26 (2002), 521-529.

[6] C. Li, C. Tsai, S. Yang, Analysis of epidemic spreading of an SIRS model in complex heterogeneous networks, Commun. Nonlinear Sci. Numer. Simul., 19 (2014), 1042-1054.

[7] L. Chen, J. Sun, Global stability and optimal control of an SIRS epidemic model on heterogeneous networks, Physica A, 10 (2014), 196-204. 
[8] J. Liu, T. Zhang, Epidemic spreading of an SEIRS model in scale-free networks, Commun. Nonlinear Sci. Numer. Simul., 16 (2011), 3375-3384.

[9] J. Zhang, Z. Jin, The analysis of an epidemic model on network, Appl. Math. Comput., 217 (2011), 7053-7064.

[10] J. Zhao, M. Liu, W. Wang, P. Yang, The stability of SI epidemic model in complex networks with stochastic perturbation, Abstr. Appl. Anal., (2014), Art. ID 610959.

[11] S. Bonaccorsi, S. Ottaviano, Epidemics on networks with heterogeneous population and stochastic infection rates, Math. Biosci., 279 (2016), 4352 .

[12] Y. Zhang, Y. Li, Evolutionary dynamics of stochastic SEIR models with migration and human awareness in complex networks, Complexity, (2020), Art. ID 3768083.

[13] M. Li, Z. Shuai, Global-stability problem for coupled systems of differential equations on networks, J. Differ. Equ., 248 (2010), 1-20.

[14] H. Guo, M. Li, Z. Shuai, A graph-theoretic approach to the method of global Lyapunov functions, Pan Am. Math. Soc., 136 (2008), 2793-2802.

[15] T. Kuniya, Y. Muroya, Global stability of a multi-group SIS epidemic model with varying total populationsize, Appl. Math. Comput., 256 (2015), 785-798. 